\title{
PNPase is a key player in the regulation of small RNAs that control the expression of outer membrane proteins
}

\author{
JOSÉ M. ANDRADE and CECÍLIA M. ARRAIANO \\ Instituto de Tecnologia Química e Biológica, Universidade Nova de Lisboa, 2781-901 Oeiras, Portugal
}

\begin{abstract}
In this report, we demonstrate that exonucleolytic turnover is much more important in the regulation of sRNA levels than was previously recognized. For the first time, PNPase is introduced as a major regulatory feature controlling the levels of the small noncoding RNAs MicA and RybB, which are required for the accurate expression of outer membrane proteins (OMPs). In the absence of PNPase, the pattern of OMPs is changed. In stationary phase, MicA RNA levels are increased in the PNPase mutant, leading to a decrease in the levels of its target ompA mRNA and the respective protein. This growth phase regulation represents a novel pathway of control. We have evaluated other ribonucleases in the control of MicA RNA, and we showed that degradation by PNPase surpasses the effect of endonucleolytic cleavages by RNase E. RybB was also destabilized by PNPase. This work highlights a new role for PNPase in the degradation of small noncoding RNAs and opens the way to evaluate striking similarities between bacteria and eukaryotes.
\end{abstract}

Keywords: MicA RNA; ompA; PNPase; RybB RNA; small RNA

\section{INTRODUCTION}

Small noncoding RNAs (sRNAs) have very important regulatory roles, and many sRNAs can be induced by changes in the environmental conditions (Storz et al. 2005). MicA and RybB RNAs are stationary phase regulators and belong to the emerging group of sRNAs that control outer membrane permeability. RybB RNA controls the expression of two outer membrane proteins, OmpC and OmpW, while MicA RNA specifically controls the expression of OmpA, an abundant outer membrane protein that has an important structural role and is involved in pathogenesis (Guillier et al. 2006). Both sRNAs act as antisense RNA and bind the 5' UTR of their target mRNAs, inhibiting translation. Moreover, MicA triggers the decay of ompA mRNA in an Hfq-dependent manner (Rasmussen et al. 2005; Udekwu et al. 2005; Johansen et al. 2006). Recent studies have described their transcriptional regulation and established MicA and RybB RNAs as new members of the $\sigma^{\mathrm{E}}$-regulon (Figueroa-Bossi et al. 2006; Johansen et al. 2006; Papenfort et al. 2006; Udekwu and Wagner 2007).

Reprint requests to: Cecília M. Arraiano, Instituto de Tecnologia Química e Biológica, Universidade Nova de Lisboa, Apartado 127, 2781901 Oeiras, Portugal; e-mail: cecilia@itqb.unl.pt; fax: (+351) 214411277. Article published online ahead of print. Article and publication date are at http://www.rnajournal.org/cgi/doi/10.1261/rna.683308.
However, nothing is known about the post-transcriptional control of either MicA or RybB RNA, and this can be determinant in controlling the levels of these sRNAs.

For the sRNAs that have been analyzed, endonucleolytic processing seems to be an important regulatory event. RNase $\mathrm{E}$ has been suggested to be the main endonuclease, although others like RNase III can also be involved (Wassarman et al. 1999). RNase II and PNPase are major Escherichia coli $3^{\prime}-5^{\prime}$ exonucleases, and a double mutant is not viable (Donovan and Kushner 1986; Régnier and Arraiano 2000). PNPase is a phosphate-dependent exonuclease that acts as a trimer (Pruijn 2005). The recent available structure of RNase II highlighted the mechanism of degradation of this hydrolytic enzyme (Amblar et al. 2006, 2007; Frazão et al. 2006; Zuo et al. 2006). Homologs of these exonucleases exist in eukaryotic exosomes (a multiprotein complex of exonucleases), and they were shown to be essential in the processing of noncoding RNAs (Houseley et al. 2006). Some sRNAs can also require 3' exonucleolytic trimming to become functional, and exonucleases can play essential roles in RNA quality control ( $\mathrm{Li}$ et al. 1998, 2002; Cairrão et al. 2003). Recently, it was demonstrated that bacterial exonucleolytic degradation can also proceed in the $5^{\prime}-3^{\prime}$ direction (Mathy et al. 2007).

In this work, we have investigated the post-transcriptional regulation of MicA RNA. We have shown that PNPase mediated degradation can be an essential regulatory 
feature in the expression of a small RNA, and as a result affect its target mRNA with consequences at the protein level. The stabilization of the MicA RNA in the PNPase mutant is higher than the one obtained in an RNase E mutant. Furthermore, we have also shown that PNPase controls the levels of RybB, another chromosomal encoded sRNA also controlling outer membrane protein expression. Here, we demonstrate that exonucleolytic turnover is a much more important feature in the control of sRNA levels than was previously recognized.

\section{RESULTS}

\section{PNPase regulates ompA expression and MicA RNA levels}

We have previously shown that exonucleolytic digestion is very important in the control of ompA transcript levels (Andrade et al. 2006). We have found that the levels of ompA mRNA were drastically reduced in the absence of PNPase (Fig. 1A), even though it is possible to observe a band using much higher amounts of total RNA (Andrade et al. 2006). The observed differences in ompA expression could be related to changes in the amount of the regulatory
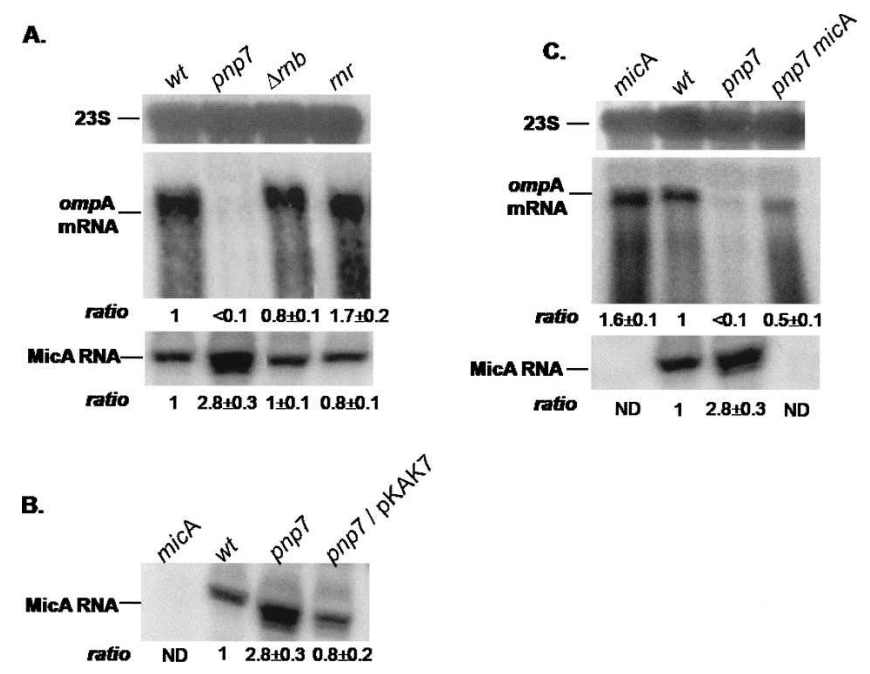

FIGURE 1. PNPase regulates ompA and MicA RNA levels. (A) Analysis of ompA mRNA and MicA RNA levels in the wild type and exonuclease mutant strains in stationary phase. ompA mRNA was analyzed by agarose Northern blot. (Upper panel) Reprobing the same membrane with a 23S-RNA probe. (Lower panel) MicA RNA expression was analyzed with the same RNAs in a polyacrylamide Northern. (B) Northern blot showing that overexpression of PNPase from plasmid pKAK7 complements the pnp mutant and changes MicA RNA levels to wild-type amounts. (C) Analysis of PNPase and MicA absence in the levels of ompA mRNA in stationary phase cells. (Top) Loading control with a $23 \mathrm{~S}$ RNA probe. (Bottom) MicA RNA expression was analyzed as an additional control. Full-length transcripts were quantified using a Molecular Dynamics PhosphorImager. The amount of the RNA found in wild type was set as 1 . The ratio between each strain and the wild type is depicted. (ND) Nondetectable.
MicA RNA. To test this, we have analyzed its expression in different ribonuclease deficient strains. MicA RNA levels were shown to be up-regulated (a threefold increase) in the absence of PNPase and were not altered by any of the other exonucleases analyzed (Fig. 1A). In addition, overexpression of PNPase from a plasmid complemented the $p n p$ mutant and reverted MicA RNA levels to wild-type (wt) condition (Fig. 1B). The above results implicate PNPase in the control of MicA RNA concentration in the cell.

To evaluate if it was possible to establish a direct correlation between the increasing levels of MicA and the reduced quantity of ompA mRNA in the absence of PNPase, we decided to compare micA and $p n p$ mutants. As expected, a higher concentration of ompA mRNA was found in micA cells. Moreover, the combined absence of $p n p$ and micA mutations results in a significant increase in ompA transcripts when compared with the pnp single mutant (Fig. 1C), revealing a direct role between MicA levels and the reduction of ompA mRNA levels in pnp7 cells. Nevertheless, in the double mutant $p n p$ micA, the ompA mRNA levels are $\sim 50 \%$ of the wild-type levels. This shows that in the absence of PNPase, MicA is probably not the only regulator of ompA expression. In fact, it has been recently described that ompA is also regulated by RseX (Douchin et al. 2006).

\section{Outer membrane composition is altered in a pnp mutant strain}

The above results prompted us to analyze if the reduced levels of ompA mRNA found in the absence of PNPase had consequences at the protein level. For this purpose, we analyzed total protein extracts from stationary phase cultures of the wild-type and the pnp mutant strain by $2 \mathrm{D}$ gel electrophoresis (Fig. 2A). When compared with wild type, the spot corresponding to OmpA is less intense in the pnp mutant. Thus, the results showed that OmpA levels are reduced in the absence of PNPase and established a parallel between ompA mRNA and OmpA protein levels. It was previously reported that PNPase activity was important for cell membrane-associated processes, but the reasons for this were unclear (Nicholson 1999). To further elucidate if the outer membrane composition was being generally affected by PNPase, or rather if it was specific to OmpA, we analyzed the outer membrane protein (OMP) profile of wild-type and $p n p$ mutant strains. It is possible to observe a distinct pattern of OMPs between stationary phase cultures of these strains (Fig. 2B). Besides the reduction in OmpA levels, the pnp mutant also changed the expression of other membrane proteins.

\section{PNPase is involved in the growth phase regulation of the antisense MicA RNA}

We have monitored the degradation of the MicA RNA in wild-type and exonuclease mutant cells in stationary phase. 
A.

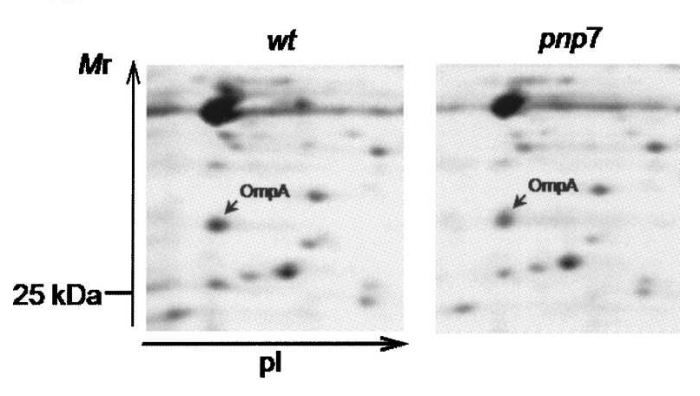

B.

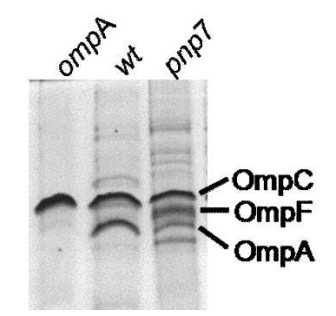

FIGURE 2. Protein profile of Escherichia coli MG1693 (wt) and SK5691 ( $p n p 7)$ strains from stationary phase cultures. (A) Twodimensional gel electrophoresis (2D-PAGE) analysis of total protein extracts. Proteins with $\mathrm{pI}$ values in the range 3.0-10.0 and molecular mass in the range of $10-250 \mathrm{kDa}$ were resolved in $12 \%$ SDS-PAGE gels followed by Coomassie G-250 staining; corresponding sections of representative gels show the down-regulation of the OmpA porin in the $p n p 7$ strain compared with wild type. (Arrow) OmpA spot. (B) Outer membrane protein fraction analysis by urea-SDS-polyacrylamyde gel electrophoresis. The positions of the OmpC, OmpF, and OmpA bands are indicated. An ompA mutant was used as control.

Strikingly, the pnp mutant showed more than a threefold increase (from 8.2-27.5 min) in full-length MicA RNA stability when compared with wild type (Fig. 3A,B). This agreed with the threefold increase in the steady-state levels of MicA RNA. The other two main exonucleases, RNase II and RNase R, did not affect the stability of the MicA RNA. Moreover, PNPase was also shown to be important in the decay of degradation intermediates. Only in this mutant can we observe increased stabilization of these smaller species of RNA (Fig. 3A). Furthermore, PNPase degradation of the MicA RNA was shown to be a stationary-phasespecific mechanism, and exponentially growing cells did not present this regulation (Fig. 3C,D). Neither RNase II nor RNase R was shown to significantly affect the stability of this sRNA, independently of the growth phase. Contrastingly, MicA RNA levels are found to be down-regulated in the absence of PNPase in the exponential phase. However, RNA stability is not significantly changed. This could possibly indicate an indirect role for PNPase in the transcriptional control of MicA RNA in the exponential phase. Altogether, these results showed that post-transcriptional regulation by PNPase is a main feature controlling MicA expression.
The role of PNPase on MicA RNA decay is independent of the target ompA mRNA

It is known that MicA acts as an antisense RNA, which binds to the 5' UTR of its target ompA transcript in an Hfqdependent fashion, promoting mRNA decay by RNase E (Rasmussen et al. 2005; Udekwu et al. 2005). To clarify if PNPase-mediated degradation of MicA RNA was dependent on the binding of the small RNA to its target ompA mRNA, we examined the decay of MicA in the absence of ompA. We have generated an ompA deletion mutant, and we also constructed the double mutant pnp ompA. Results showed that the absence of its target ompA mRNA does not significantly affect MicA RNA stability (Fig. 4). Moreover, PNPase-mediated degradation of MicA RNA is shown to be independent of the presence of ompA mRNA, as the double mutant $p n p$ ompA showed identical stability to the pnp single mutant. Consequently, PNPase does not require the previous binding of the MicA RNA to its target ompA mRNA for efficient degradation of this antisense RNA.

\section{Polyadenylation participates in the decay of the MicA RNA}

Polyadenylation is a major regulatory process controlling gene expression (Régnier and Arraiano 2000; Dreyfus and Régnier 2002). Polyadenylation of some sRNAs has been described, and polyadenylated RNA is a better substrate for PNPase (Xu and Cohen 1995; Lisitsky and Schuster 1999). To address whether PNPase was acting through a Poly(A)dependent pathway in the decay of the MicA RNA, we first studied the pcnB mutant strain, which is defective in PAP I, responsible for $>90 \%$ of the polyadenylation in E. coli (O'Hara et al. 1995). We have found a slight increase in the half-life in the absence of polyadenylation (Fig. 5), revealing the involvement of PAP I in the MicA RNA degradation pathways. We have also analyzed MicA RNA decay in the $p n p \quad \Delta p c n \mathrm{~B}$ double mutant. Results showed that the combined absence of PNPase and PAP I presented about the same MicA RNA stability as found in the single absence of PNPase. These results indicated that polyadenylation by PAP I appears not to be critical for PNPase activity on the MicA RNA turnover.

\section{MicA is more stabilized in the absence of PNPase than in the RNase $E$ mutant}

RNase E is an important enzyme controlling sRNA stability (Wassarman et al. 1999; Moll et al. 2003; Morita et al. 2005; Suzuki et al. 2006). Therefore, we have also analyzed the role of RNase $\mathrm{E}$ in the degradation of MicA RNA in stationary phase cells. Since RNase E is an essential enzyme, we used the rnel thermosensitive allele (Arraiano et al. 1988). Wild-type and $p n p$ mutant strains were studied in 
A.
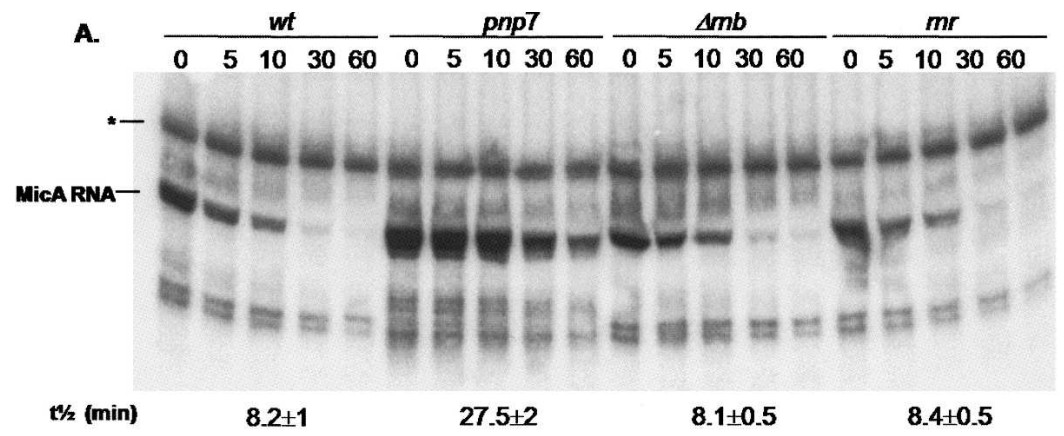

c.

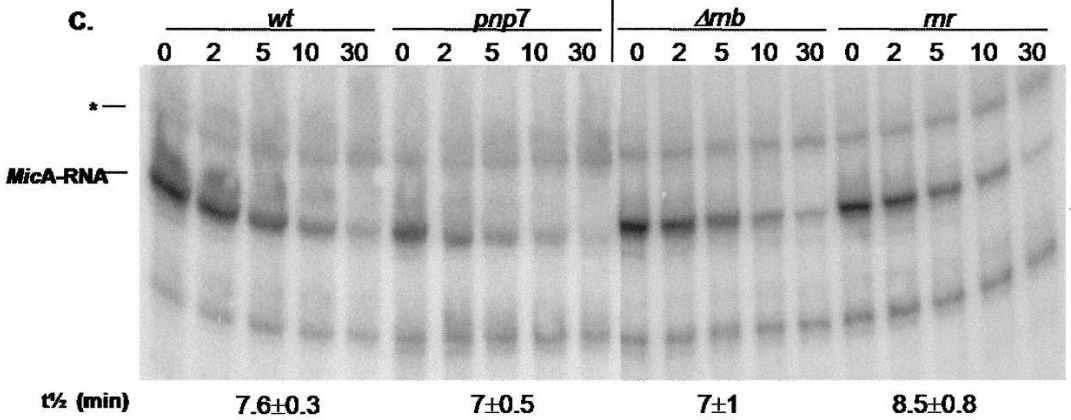

B.

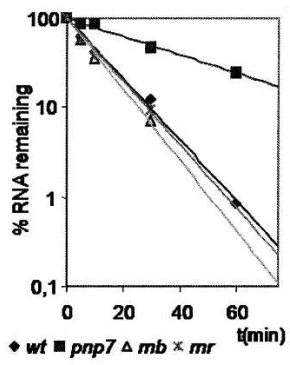

D.

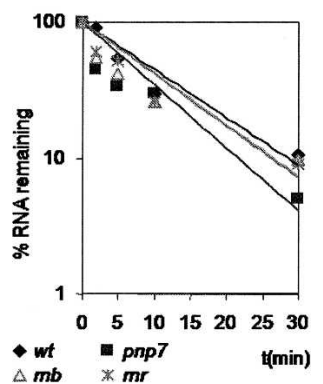

FIGURE 3. Comparison of the decay rates of the MicA RNA in the wild-type and exonuclease mutants in stationary and exponential phase. $(A)$ Northern blot of RNA extracted from stationary phase cultures. $(C)$ Northern blot of RNA extracted from exponential phase cultures. Total RNA was extracted from culture samples withdrawn after inhibition of transcription (timepoints are shown in minutes). $\left(^{\star}\right)$ Nonspecific band that cross-reacts with the antisense MicA-probe. A more stringent washing eliminates this band, while the signal for MicA RNA remains. (B,D) The band corresponding to the full-length MicA RNA was quantified with a PhosphorImager and plotted versus time of extraction (min).

the same conditions. Results shown in Figure 6, A and B, demonstrated that RNase $\mathrm{E}$ is involved in the decay of the full-length MicA RNA (a twofold increased stability is observed in the rne1 mutant when compared with wild type). Comparatively, the $p n p$ mutant presented a higher stabilization of this sRNA (more than a threefold increase). Analysis of the double mutant rne 1 pnp showed the highest stability for MicA RNA (more than a 10-fold increase), greater than any of the stabilities found in the single mu- tants. This large stability indicates that PNPase and RNase $\mathrm{E}$ are major factors involved in the MicA RNA turnover.

\section{The degradosome assembly is not crucial for PNPase-mediated degradation of the MicA RNA}

PNPase and RNase E can physically interact in the degradosome, a multiprotein complex involved in mRNA
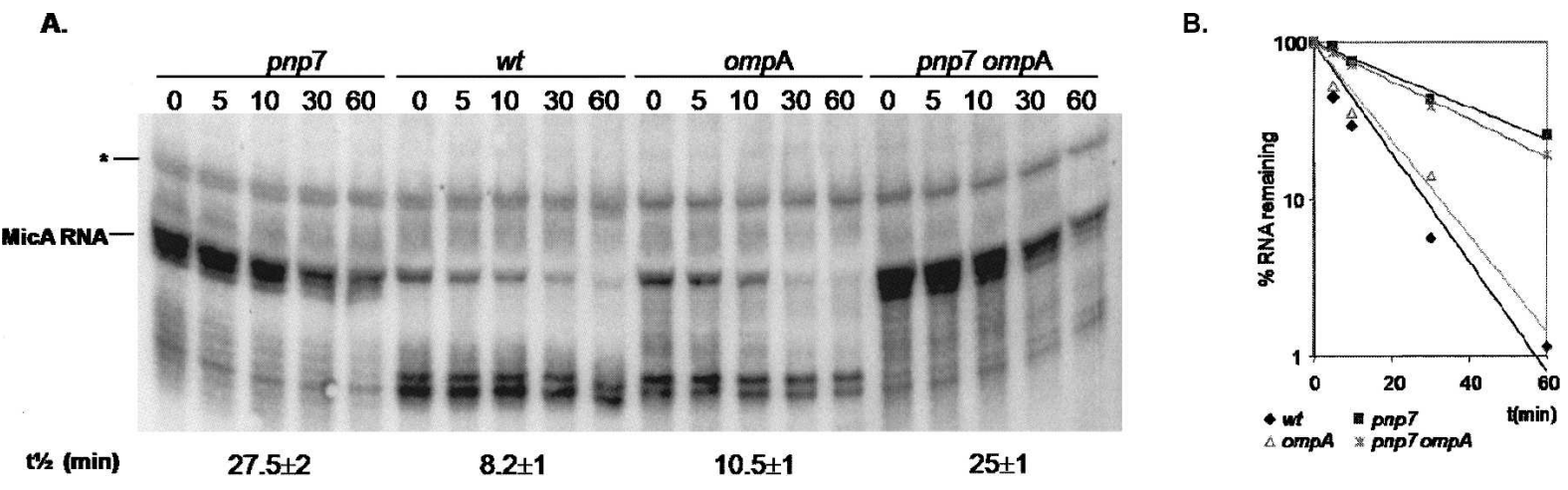

FIGURE 4. MicA RNA degradation in the absence of the target ompA mRNA. (A) Northern blot comparing the importance of ompA mRNA in the decay rate with its antisense regulator MicA RNA. Timepoints of sample cultures are shown in minutes after inhibition of transcription. $\left({ }^{*}\right)$ Nonspecific cross-hybridization with the antisense MicA-probe. (B) Full-length MicA RNA stability, after PhosphorImager quantification along time of sample extraction (in min). 


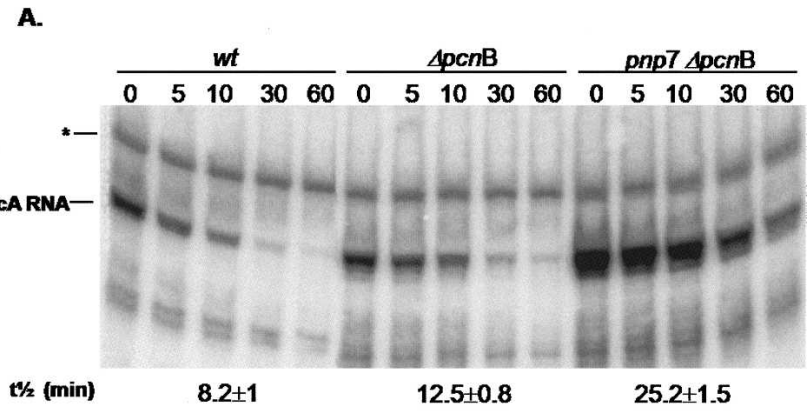

B.

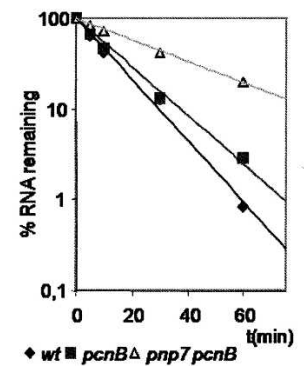

FIGURE 5. The role of the polyadenylation in MicA RNA decay in stationary phase. (A) The MicA RNA stability in the wild type and in the absence of PAP I-dependent polyadenylation $(\Delta p c n \mathrm{~B}$ mutation) was verified by Northern blot analysis. MicA RNA half-lives are shown in minutes $(\mathrm{min}) .\left(^{*}\right)$ Nonspecific band that was used as loading control. (B) Relative amount of the full-length MicA RNA remaining at each timepoint (as determined by PhosphorImager analysis) plotted as function of time.

degradation (Carpousis 2002). To determine the importance of this association in MicA RNA degradation, we performed RNA decay experiments with the rne131 mutant (that is impaired in degradosome assembly) (Fig. 6C). In the absence of the degradosome, MicA RNA presented a more than twofold stabilization. However, the pnp single mutant presents a higher stabilization, revealing that the role of PNPase exceeds the role of the degradosome activity. In fact, the double mutant rne131 pnp showed an increased stability when compared with the rne131 mutant (Fig. 6C,D). These results confirmed that PNPase can act in this sRNA in a degradosome-independent manner.

\section{The noncoding RybB RNA is under the control of PNPase}

The results described above raised the question if the effect of PNPase was particular to MicA or whether it could be extended to other sRNAs. We have evaluated the stability of the small RybB in both the wild-type and pnp mutant strains. RybB acts as an antisense RNA and regulates the expression of both membrane proteins OmpC and OmpW in stationary phase (Guillier et al. 2006; Johansen et al. 2006). We have shown that RybB is accumulated in the absence of PNPase, and that this is due to an increasing stability found in the $p n p$ mutant (Fig. 7). Moreover, PNPase is also shown to be important for the degradation of breakdown products. As observed, also smaller species of this sRNA are strongly accumulated in the absence of PNPase. These results emphasize the role of PNPase in the control of sRNAs levels and outer membrane protein expression in stationary phase.

\section{DISCUSSION}

We have shown here that $3^{\prime}$ exonucleolytic degradation is more important in the regulation of sRNA levels than was previously recognized. Transcription was shown to be important in the control of MicA RNA levels that are increased in stationary phase (Figueroa-Bossi et al. 2006; Johansen et al. 2006; Papenfort et al. 2006; Udekwu and Wagner 2007). Here, we have shown for the first time that the growth phase regulation of MicA RNA is highly dependent on post-transcriptional control. Most interestingly, from the major exonucleases involved in mRNA decay, only PNPase was shown to be important in the control of MicA. During stationary phase, the level of MicA RNA was significantly higher in cells containing a mutant allele of the pnp gene, which encodes the $3^{\prime}$ exonuclease polynucleotide phosphorylase. Moreover, this increase in the level of MicA was associated with a decrease in the cellular level of the target ompA mRNA and the OmpA protein. Assay of the half-life of MicA showed that this RNA was more stable (about threefold) in the pnp mutant. This result provided an explanation for the increase in MicA level and indicated that this RNA is a substrate for PNPase.

Previous studies in which MicA was overproduced from a plasmid have demonstrated that an increase in the level of this RNA correlates with the down-regulation of ompA mRNA (Rasmussen et al. 2005; Udekwu et al. 2005). We also confirmed that the reduction in the ompA mRNA level in the pnp mutant was dependent to a large extent on the presence of MicA. Given the above findings, it seems likely that the inactivation of PNPase will impact the levels of other MicA targets such as the mRNA of LamB, recently reported in Salmonella (Bossi and Figueroa-Bossi 2007). While the level of ompA mRNA increased in a pnp mutant background when MicA was removed, it was not restored to the level in wild-type cells. This indicates that there is a second, MicA-independent pathway by which PNPase can influence the levels of ompA mRNA. RseX, one other sRNA implicated in ompA expression, is thus a good candidate to be also regulated by PNPase (Douchin et al. 2006).

Interestingly, PNPase affected MicA when cells were in the stationary phase, but it did not change MicA stability in exponential phase cultures. This indicates that the PNPasemediated decay of MicA RNA is growth-phase dependent. It has also been reported that PNPase is the only $3^{\prime}$ exonuclease involved in the degradation of certain mRNAs in stationary phase in Streptococcus (Barnett et al. 2007). 
A.

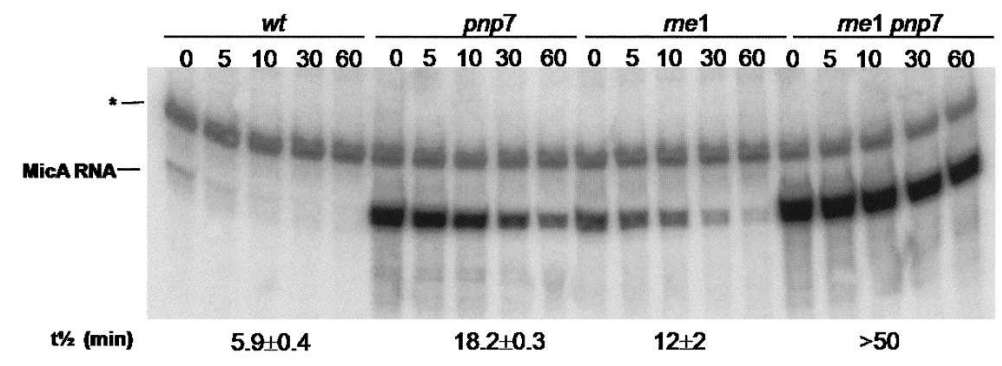

c.

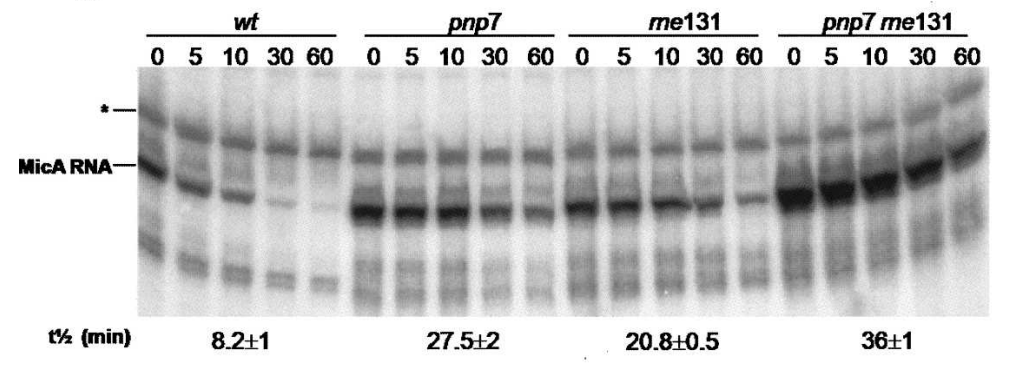

B.

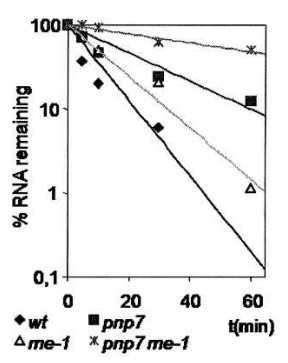

D.

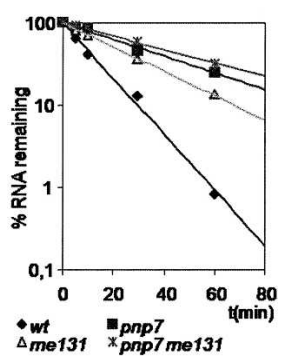

FIGURE 6. RNase $\mathrm{E}$ is involved in the control of the MicA RNA stability. ( $A$ ) Cultures were grown at $30^{\circ} \mathrm{C}$ to an $\mathrm{OD}_{600} \approx 2.0$ and then shifted to the nonpermissive temperature of $44^{\circ} \mathrm{C}$. After $5 \mathrm{~min}$, transcription was blocked with the addition of rifampicin, and samples were withdrawn at the times indicated. Total RNA was extracted as described in the Materials and Methods. MicA RNA stability was analyzed using an antisense RNA probe. (C) RNase E mutant cells impaired in degradosome assembly (rnel31 mutation) were analyzed with wild-type and pnp mutant strains. MicA RNA stability is shown in minutes $(\min ) .\left(^{*}\right)$ Nonspecific band. $(B, D)$ Full-length MicA RNA was quantified with a PhosphorImager and plotted versus time of extraction (min).

The 3' exonucleases RNase II and RNase R appear to be not required for the normal rate of decay of MicA RNA. Hfq, a pleiotrophic regulator with very important roles on RNA (Valentin-Hansen et al. 2004), also participates in the stability of MicA (J.M. Andrade and C.M. Arraiano, unpubl.). Hfq levels are described to decrease upon entrance to stationary phase (Ali Azam et al. 1999). Thereby, it is possible that down-regulation in Hfq levels results in a more unprotected MicA RNA, allowing PNPase to effectively degrade it.

The decay of MicA appears also to be independent of ompA mRNA in backgrounds that were wild-type or mutant with regard to PNPase. Thus, while binding between the sRNA and its target mRNA has been reported to be important for the decay of sRNA (Massé et al. 2003; Afonyushkin et al. 2005), this is not the case for PNPasemediated decay of MicA. Interestingly, we have also shown that PNPase-mediated decay of MicA RNA during stationary phase is not dependent on Poly(A) Polymerase I. This may be related to the finding that PNPase can also work as a polymerase when inorganic phosphate is limiting, as can happen in stationary phase (Mohanty and Kushner 2000).

PNPase and RNase E can physically interact in the degradosome, a multiprotein complex involved in RNA turnover (Carpousis 2002), and these enzymes have been shown to interact functionally in the decay of some sRNAs such as RyhB, SgrS, and CrsB (Massé et al. 2003; Morita et al. 2005; Suzuki et al. 2006). We found that MicA was stabilized in RNase $\mathrm{E}$ mutants that were temperaturesensitive or were unable to form the degradosome. These results revealed the importance of RNase E and clearly established that degradosome in the degradation of the MicA RNA. Moreover, when both of the corresponding rne mutations were combined with a $p n p$ mutation, the level of stabilization was even higher, supporting the idea that RNase E and PNPase can act independently. Accordingly, the effect of PNPase on MicA is not exclusively due to the activity of PNPase in the degradosome.

PNPase activity on sRNAs appears to be not restricted to MicA; we found also that the small RybB is stabilized in the pnp mutant. Together with MicA, the RybB RNA is also a regulator of outer membrane protein expression (Guillier et al. 2006; Johansen et al. 2006). The notion that PNPase is a key player controlling OMP expression, namely through regulation of riboregulators stability, highlights the importance of this enzyme in RNA metabolism and unveils an important role in the adaptation to stationary phase (Fig. $8)$. The outer membrane provides the first cellular contact with the exterior, and the accurate control of outer membrane composition is essential for survival. A diversity of sRNAs was shown to play a major role on this regulation (Guillier et al. 2006). Earlier reports associate PNPase activity with cell membrane-associated processes such as 


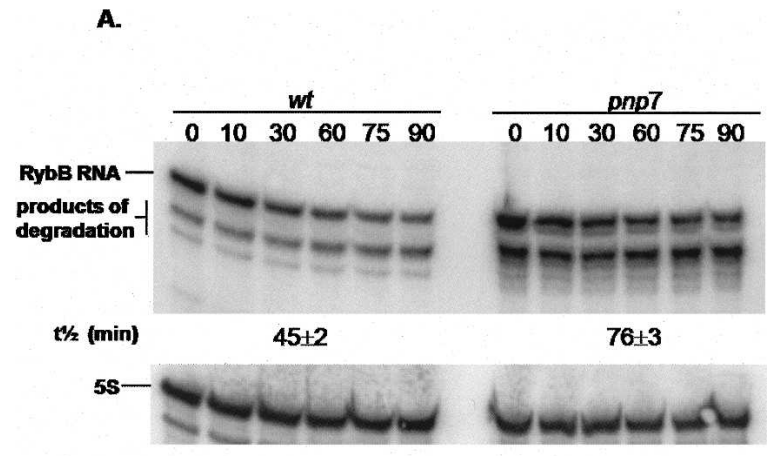

B.

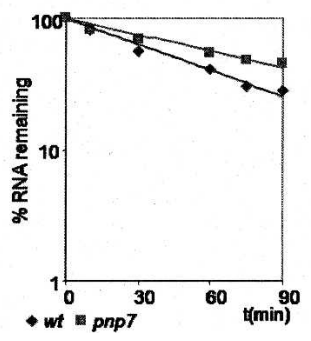

FIGURE 7. PNPase controls the small RybB RNA. (A) Northern blot analysis of the stability of the RybB RNA in the wt and $p n p$ mutant strains in stationary phase. Full-length RybB and breakdown products of degradation are observable. (Lower panel) The same membrane was stripped and rehybridized with a 5S RNA riboprobe as a loading control. $(B)$ The signal corresponding to the full-length RybB RNA was quantified with a PhosphorImager and plotted versus time of extraction ( $\mathrm{min})$.

antibiotic uptake (McMurry and Levy 1987; Nicholson 1999). We now show that PNPase-mediated degradation of small RNAs controlling outer membrane protein expression can change the profile of OMP in the cell. This may promote membrane alterations with an impact in cellular functions such as cellular morphogenesis, permeability, or virulence.

In eukaryotes, the exosome (a complex of exonucleases reminiscent of PNPase) was shown to be essential in the maturation of noncoding RNAs (Houseley et al. 2006). We envisage that PNPase may be involved in the degradation of other small RNAs, making PNPase-mediated decay a more general feature of stable RNA metabolism.

\section{MATERIALS AND METHODS}

\section{Bacterial strains and plasmids}

The strains used in this work are indicated in Table 1. All experiments were performed in the E. coli MG1693 (wild type) and its derivative isogenic strains. Strain $\Delta o m p A:$ cam was constructed using a one-step inactivation system of chromosomal genes (Datsenko and Wanner 2000). Deletion of the ompA gene completely eliminates the binding site for the antisense MicA RNA. After transformation with the PCR fragment containing the ompA

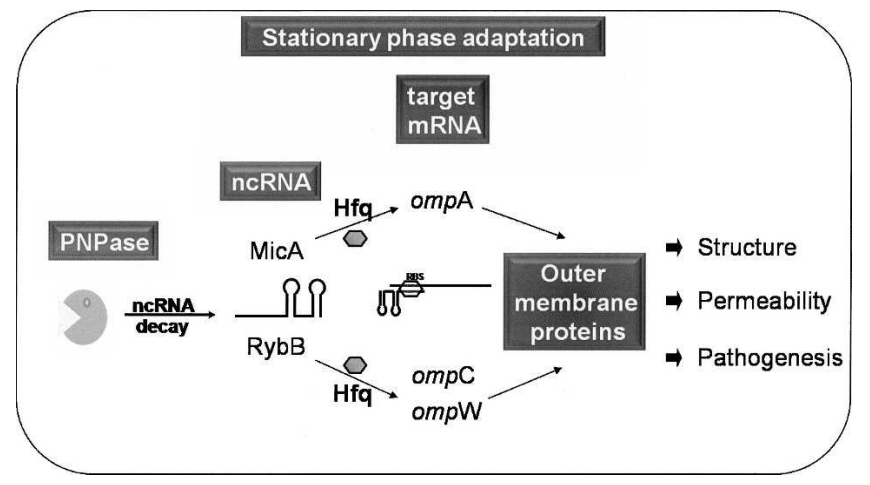

FIGURE 8. PNPase-mediated degradation is a major regulatory event controlling the levels of sRNAs (namely MicA and RybB) that are required for the accurate expression of outer membrane proteins. These sRNAs act as antisense RNAs and bind to the $5^{\prime}$ UTR of their target outer membrane mRNAs in an Hfq-dependent mechanism. This process inhibits translation and can help promoting the decay of the target mRNAs. Balanced outer membrane protein composition is essential for survival and affects many cellular processes, such as morphology, permeability, and virulence. PNPase emerges as an important enzyme in the growth phase adaptation to stationary phase.

deletion into the MG1693 strain, chloramphenicol-resistant colonies were isolated. Transformants were reisolated on selective plates, and mutation was reintroduced into MG1693. The mutations were transferred by P1vir transduction as described (Cairrão et al. 2003). Plasmid pKAK7 was described elsewhere (Donovan and Kushner 1986). Primers used in this work are listed in Table 2.

\section{Growth conditions}

Bacteria were grown at $37^{\circ} \mathrm{C}$ unless stated otherwise, in LuriaBertani (LB) medium supplemented with thymine $(50 \mu \mathrm{g} / \mathrm{mL})$. Growth was performed as described (Andrade et al. 2006). When required, antibiotics were present at the following concentrations: chloramphenicol, $50 \mu \mathrm{g} / \mathrm{mL}$; kanamycin, $50 \mu \mathrm{g} / \mathrm{mL}$; tetracycline, $20 \mu \mathrm{g} / \mathrm{mL}$; ampicillin, $150 \mu \mathrm{g} / \mathrm{mL}$.

TABLE 1. Bacterial strains used in this study

\begin{tabular}{lll}
\hline Strain & \multicolumn{1}{c}{ Relevant genotype } & \multicolumn{1}{c}{ Reference } \\
\hline G897 & MC4100 relA $A^{+}$micA & Udekwu et al. (2005) \\
HM104 & thyA715 rnr & Cairrão et al. (2003) \\
MG1693 & thyA715 & Arraiano et al. (1988) \\
SK5665 & thyA715 rne1 & Arraiano et al. (1988) \\
SK5671 & thyA715 rne1 pnp7 & Arraiano et al. (1988) \\
SK5691 & thyA715 pnp7 & Arraiano et al. (1988) \\
SK7988 & thyA715 $\Delta p c n B$ & O'Hara et al. (1995) \\
CMA201 & thyA715 rnb & Cairrão et al. (2003) \\
CMA300 & thyA715 pnp7 $\Delta p c n B$ & This study \\
CMA413 & thyA715 micA & This study \\
CMA414 & thyA715 pnp7 micA & This study \\
CMA418 & thyA715 pnp7 rne131 & This study \\
CMA440 & thyA715 pnp7 / pKAK7 & This study \\
CMA445 & thyA715 ompA & This study \\
CMA446 & thyA715 pnp7 $\Delta$ ompA & This study \\
CMA501 & thyA715 rne131 & Viegas et al. (2005) \\
\hline
\end{tabular}


TABLE 2. Primers used in this study

\begin{tabular}{|c|c|c|}
\hline Primer & Sequence & Description \\
\hline micA-T7 & 5'-TAATACGACTCACTATAGGAAGGCCACTCGTGAGTGGCCAA-3' & MicA RNA antisense probe \\
\hline micA-F & 5'-GAAAGACGCGCATTTGTTATC-3' & \\
\hline micA1 & 5'-TTTTCGCCACCCGAACTGCAGGC-3' & Confirmation of micA deletion \\
\hline micA2 & 5'-AATGGAAGCTTCTGATACCGAACCG-3' & \\
\hline ompA-T7 & 5'-TAATACGACTCACTATAGGAAAAAAAACCCCGCAGCAGC-3' & ompA RNA antisense probe \\
\hline ompA-F & 5'-TTGTAGACTTTACATCGCCAGGG-3' & \\
\hline ompAdelF & $\begin{array}{l}\text { 5'-CAGACGAGAACTTAAGCCTGCGGCTGAGTTACAACGTCTTTGATAC } \\
\text { CTTTAACGTGTAGGCTGGAGCTGCTTC-3' }\end{array}$ & ompA deletion \\
\hline ompAdelR & $\begin{array}{l}\text { 5'-TTGTCGCTGACAAAAAAGATTAAACATACCTTATACAAGACTTTTTTT } \\
\text { TCGGTCCATATGAATATCCTCCTTAG-3' }\end{array}$ & \\
\hline ompA1 & 5'-CCCAGCCAATGCTTTCAGTCGTGAC-3' & Confirmation of ompA deletion \\
\hline ompA2 & 5'-ACGCGCAAAAAATCGATCGATCTGG-3' & \\
\hline rybB-T7 & 5'-TAATACGACTCACTATAGGAACAAAAAACCCATCAACCTTGAACCG-3' & RybB RNA antisense probe \\
\hline rybB-F & 5'-ACTGCTTTTCTTTGATGTCCC-3' & \\
\hline 5S-RNA-F & 5'-AAACAGAATTTGCCTGGCGGCAGTAG-3' & 5S-RNA probe \\
\hline 5S-RNA-R & 5'-IAATACGACTCACTATAGGATGCCTGGCAGTTCCСТАCTCTCGC-3' & \\
\hline 23S-RNA-F & 5'-TGTGAGGTTAAGCGACTAAGC-3' & 23S-RNA probe \\
\hline 23S-RNA-R & 5'-TTCGGGGAGAACCAGCTATC-3' & \\
\hline
\end{tabular}

\section{RNA extraction and Northern blot analysis}

Total RNA was extracted as previously described (Andrade et al. 2006). Ten micrograms of total RNA from each strain was used to analyze ompA mRNA on a $1.2 \%$ agarose MOPS/formaldehyde gel. RNA was transferred to Hybond- $\mathrm{N}^{+}$membrane (Amersham Biosciences) by capillarity using $20 \times$ SSC as a transfer buffer. Thirty micrograms of total RNA was used to analyze MicA RNA on $10 \%$ polyacrylamide gels. RNA was electrotransferred onto Hybond- $\mathrm{N}^{+}$membrane using $1 \times$ TAE. RNA was UV cross-linked to the membrane. A DNA template from PCR obtained with Taq DNA Polymerase (Fermentas) was used to generate the riboprobes by in vitro transcription with T7 RNA Polymerase (Promega) in the presence of $\left[\alpha-{ }^{32} \mathrm{P}\right] \mathrm{UTP}$ (GE Healthcare). All riboprobes were purified on G50 Microspin columns (GE Healthcare). For loading control, the membranes used to detect ompA were stripped and rehybridized with a 23S-RNA probe. Hybridizations were carried out at $68^{\circ} \mathrm{C}$ with the PerfectHyb Plus Hybridization Buffer (Sigma).

\section{RNA half-life determination}

Quantification of full-length RNA was done after PhosphorImager scanning by the IMAGEQUANT program (Molecular Dynamics). The half-lives of RNA were determined by linear regression using the logarithm of the percentage of RNA remaining versus time, considering the amount of RNA at $0 \mathrm{~min}$ as $100 \%$. The data presented are the average of different Northern blots (two to 10) from at least two independent RNA extractions.

\section{Protein analysis}

The protocol for the two-dimensional gel electrophoresis experiments was done as described with modifications (Andrade et al. 2006). Two hundred micrograms of total protein extracts (after precipitation with TCA 10\%/acetone) was soluble in $250 \mu \mathrm{L}$ of rehydration buffer (7 M urea, $2 \mathrm{M}$ thiourea, 2\% CHAPS, 0.5\% [v/v] IPG buffer [GE Healthcare], $50 \mathrm{mM} \mathrm{DTT).}$
First-dimension electrophoresis was carried on linear immobilized pH 3-10 gradient (IPG) strips (13 cm, Immobiline DryStrip, GE Healthcare). The rehydration step and electrophoresis were performed in an IPGphor Isoelectric Focusing Unit (Pharmacia Biotech). Following a rehydration step at $30 \mathrm{~V}$ for $12 \mathrm{~h}$, isoelectric focusing (IEF) was carried out for $32,000 \mathrm{Vhr}$, at a maximum of 8000 V. After IEF, the IPGs were either separated immediately for second dimension or kept at $-80^{\circ} \mathrm{C}$ until use. The equilibrated IPGs were separated on equal 12\% SDS-PAGEs using a Laemmi system. Gels were stained with Coomassie Brilliant Blue G-250. OmpA protein was identified based on the protein pattern (Andrade et al. 2006). Image acquisition from the gels was done using an ImageScanner (Amersham Biosciences) and the LabScan 5.0 software. The membrane protein fraction, from stationary phase cultures, was extracted as described (Matsuyama et al. 1984). Equal amounts of protein were run on a $12 \%$ SDSpolyacrylamide gel with $8 \mathrm{M}$ urea. Gels were stained overnight with Coomassie R-250.

\section{ACKNOWLEDGMENTS}

We thank Professor Gerhart Wagner for providing the micA strain and Professor Kenneth McDowall for critical reading of the manuscript. Sandra Viegas is acknowledged for helpful discussions, Rita Francisco for her valuable help with 2-DE experiments, and Vânia Pobre for technical assistance. J.M.A. is a recipient of a FCT Doctoral Scholarship. The work at ITQB was supported by Fundação para a Ciência e Tecnologia (FCT), Portugal.

Received June 12, 2007; accepted October 24, 2007.

\section{REFERENCES}

Afonyushkin, T., Večerek, B., Moll, I., Bläsi, U., and Kaberdin, V.R. 2005. Both RNase E and RNase III control the stability of $\operatorname{sodB}$ 
mRNA upon translational inhibition by the small regulatory RNA RyhB. Nucleic Acids Res. 33: 1678-1689. doi: 10.1093/nar/gki313.

Ali Azam, T., Iwata, A., Nishimura, A., Ueda, S., and Ishihama, A. 1999. Growth phase-dependent variation in protein composition of the Escherichia coli nucleoid. J. Bacteriol. 181: 6361-6370.

Amblar, M., Barbas, A., Fialho, A.M., and Arraiano, C.M. 2006. Characterization of the functional domains of Escherichia coli RNase II. J. Mol. Biol. 360: 921-933.

Amblar, M., Barbas, A., Gomez-Puertas, P., and Arraiano, C.M. 2007. The role of the S1 domain in exoribonucleolytic activity: Substrate specificity and multimerization. RNA 13: 317-327.

Andrade, J.M., Cairrão, F., and Arraiano, C.M. 2006. RNase R affects gene expression in stationary phase: Regulation of ompA. Mol. Microbiol. 60: 219-228.

Arraiano, C.M., Yancey, S.D., and Kushner, S.R. 1988. Stabilisation of discrete mRNA breakdown products in ams pnp $r n b$ multiple mutants of Escherichia coli K-12. J. Bacteriol. 170: 4625-4633.

Barnett, T.C., Bugrysheva, J.V., and Scott, J.R. 2007. Role of mRNA stability in growth phase regulation of gene expression in the group A Streptococcus. J. Bacteriol. 189: 1866-1873.

Bossi, L. and Figueroa-Bossi, N. 2007. A small RNA downregulates LamB maltoporin in Salmonella. Mol. Microbiol. 65: 799-810.

Cairrão, F., Cruz, A., Hiroyuki, M., and Arraiano, C.M. 2003. Cold shock induction of RNase $\mathrm{R}$ and its role in the maturation of the quality control mediator SsrA/tmRNA. Mol. Microbiol. 50: 13491360.

Carpousis, A.J. 2002. The Escherichia coli RNA degradosome: Structure, function, and relationship in other ribonucleolytic multienzyme complexes. Biochem. Soc. Trans. 30: 150-155.

Datsenko, K.A. and Wanner, B.L. 2000. One-step inactivation of chromosomal genes in Escherichia coli K-12 using PCR products. Proc. Natl. Acad. Sci. 97: 6640-6645.

Donovan, W.P. and Kushner, S.R. 1986. Polynucleotide phosphorylase and ribonuclease II are required for cell viability and mRNA turnover in Escherichia coli K-12. Proc. Natl. Acad. Sci. 83: 120-124.

Douchin, V., Bohn, C., and Bouloc, P. 2006. Down-regulation of porins by a small RNA bypasses the essentiality of the regulated intramembrane proteolysis protease RseP in Escherichia coli. J. Biol. Chem. 281: 12253-12259.

Dreyfus, M. and Régnier, P. 2002. The poly(A) tail of mRNAs: Bodyguard in eukaryotes, scavenger in bacteria. Cell 111: 611-613.

Figueroa-Bossi, N., Lemire, S., Maloriol, D., Balbontin, R., Casadesus, J., and Bossi, L. 2006. Loss of Hfq activates the $\sigma^{\mathrm{E}}$-dependent envelope stress response in Salmonella enterica. Mol. Microbiol. 62: 838-852.

Frazão, C., McVey, C.E., Amblar, M., Barbas, A., Vonrhein, C., Arraiano, C.M., and Carrondo, M.A. 2006. Unraveling the dynamics of RNA degradation by ribonuclease II and its RNAbound complex. Nature 443: 110-114.

Guillier, M., Gottesman, S., and Storz, G. 2006. Modulating the outer membrane with small RNAs. Genes \& Dev. 20: 2338-2348.

Houseley, J., LaCava, J., and Tollervey, D. 2006. RNA-quality control by the exosome. Nat. Rev. Mol. Cell Biol. 7: 529-539.

Johansen, J., Rasmussen, A.A., Overgaard, M., and ValentinHansen, P. 2006. Conserved small noncoding RNAs that belong to the $\sigma^{\mathrm{E}}$ regulon: Role in down-regulation of outer membrane proteins. J. Mol. Biol. 364: 1-8.

Li, Z., Pandit, S., and Deutscher, M.P. 1998. 3' exoribonucleolytic trimming is a common feature of the maturation of small, stable RNAs in Escherichia coli. Proc. Natl. Acad. Sci. 95: 2856-2861.

Li, Z., Reimers, S., Pandit, S., and Deutscher, M.P. 2002. RNA quality control: Degradation of defective transfer RNA. EMBO J. 2: 11321138.

Lisitsky, I. and Schuster, G. 1999. Preferential degradation of polyadenylated and polyuridinylated RNAs by the bacterial exoribonuclease polynucleotide phosphorylase. Eur. J. Biochem. 261: 468-474.
Massé, E., Escorcia, F.E., and Gottesman, S. 2003. Coupled degradation of a small regulatory RNA and its mRNA targets in Escherichia coli. Genes \& Dev. 17: 2374-2383.

Mathy, N., Benard, L., Pellegrini, O., Daou, R., Wen, T., and Condon, C. 2007. 5' -to- $3^{\prime}$ exoribonuclease activity in bacteria: Role of RNase J1 in rRNA maturation and $5^{\prime}$ stability of mRNA. Cell 129: 681-692.

Matsuyama, S., Inokuchi, K., and Mizushima, S. 1984. Promoter exchange between $o m p \mathrm{~F}$ and $o m p \mathrm{C}$, genes for osmoregulated major outer membrane proteins of Escherichia coli K-12. J. Bacteriol. 158: 1041-1047.

McMurry, L.M. and Levy, S.B. 1987. Tn5 insertion in the polynucleotide phosphorylase ( $p n p)$ gene in Escherichia coli increases susceptibility to antibiotics. J. Bacteriol. 169: 1321-1324.

Moll, I., Afonyushkin, T., Vytvytska, O., Kaberdin, V.R., and Blasi, U. 2003. Coincident $\mathrm{Hfq}$ binding and RNase E cleavage sites on mRNA and small regulatory RNAs. RNA 9: 1308-1314.

Mohanty, B.K. and Kushner, S.R. 2000. Polynucleotide phosphorylase functions both as a $3^{\prime}-5^{\prime}$ exonuclease and a poly(A) polymerase in Escherichia coli. Proc. Natl. Acad. Sci. 97: 11966-11971.

Morita, T., Maki, K., and Aiba, H. 2005. RNase E-based ribonucleoprotein complexes: Mechanical basis of mRNA destabilization mediated by bacterial noncoding RNAs. Genes \& Dev. 19: 2176-2186.

Nicholson, A.W. 1999. Function, mechanism, and regulation of bacterial ribonucleases. FEMS Microbiol. Rev. 23: 371-390.

O’Hara, E.B., Chekanova, J.A., Ingle, C.A., Kushner, Z.R., Peters, E., and Kushner, S.R. 1995. Polyadenylylation helps regulate mRNA decay in Escherichia coli. Proc. Natl. Acad. Sci. 92: 1807-1811.

Papenfort, K., Pfeiffer, V., Mika, F., Lucchini, S., Hinton, J.C., and Vogel, J. 2006. $\sigma^{\mathrm{E}}$-dependent small RNAs of Salmonella respond to membrane stress by accelerating global omp mRNA decay. Mol. Microbiol. 62: 1674-1688.

Pruijn, G.J. 2005. Doughnuts dealing with RNA. Nat. Struct. Mol. Biol. 12: $562-564$.

Rasmussen, A.A., Eriksen, M., Gilany, K., Udesen, C., Franch, T., Petersen, C., and Valetin-Hansen, P. 2005. Regulation of ompA mRNA stability: The role of a small regulatory RNA in growth phase-dependent control. Mol. Microbiol. 58: 1421-1429.

Régnier, P. and Arraiano, C.M. 2000. Degradation of mRNA in bacteria: Emergence of ubiquitous features. Bioessays 22: 235-244.

Storz, G., Altuvia, S., and Wassarman, K.M. 2005. An abundance of RNA regulators. Annu. Rev. Biochem. 74: 199-217.

Suzuki, K., Babitzke, P., Kushner, S.R., and Romeo, T. 2006. Identification of a novel regulatory protein (CsrD) that targets the global regulatory RNAs CsrB and CsrC for degradation by RNase E. Genes \& Dev. 20: 2605-2617.

Udekwu, K.I. and Wagner, E.G. 2007. Sigma E controls biogenesis of the antisense RNA MicA. Nucleic Acids Res. 35: 1279-1288. doi: $10.1093 / \mathrm{nar} / \mathrm{gkl1} 154$.

Udekwu, K.I., Darfeuille, F., Vogel, J., Reimegard, J., Holmqvist, E., and Wagner, E.G. 2005. Hfq-dependent regulation of OmpA synthesis is mediated by an antisense RNA. Genes \& Dev. 19: 2355-2366.

Valentin-Hansen, P., Eriksen, M., and Udesen, C. 2004. The bacterial Sm-like protein Hfq: A key player in RNA transactions. Mol. Microbiol. 51: 1525-1533.

Viegas, S.C., Schmidt, D., Kasche, V., Arraiano, C.M., and Ignatova, Z. 2005. Effect of the increased stability of the penicillin amidase mRNA on the protein expression levels. FEBS Lett. 579: 5069-5073.

Wassarman, K.M., Zhang, A., and Storz, G. 1999. Small RNAs in Escherichia coli. Trends Microbiol. 7: 37-45.

$\mathrm{Xu}, \mathrm{F}$. and Cohen, S.N. 1995. RNA degradation in Escherichia coli regulated by $3^{\prime}$ adenylation and $5^{\prime}$ phosphorylation. Nature 374: 180-183.

Zuo, Y., Vincent, H.A., Zhang, J., Wang, Y., Deutscher, M.P., and Malhotra, A. 2006. Structural basis for processivity and singlestrand specificity of RNase II. Mol. Cell 24: 149-156. 

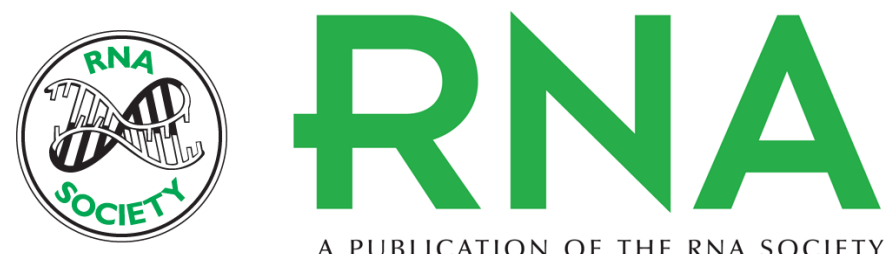

A PUBLICATION OF THE RNA SOCIETY

\section{PNPase is a key player in the regulation of small RNAs that control the expression of outer membrane proteins}

José M. Andrade and Cecília M. Arraiano

RNA 2008 14: 543-551

References This article cites 44 articles, 18 of which can be accessed free at:

http://rnajournal.cshlp.org/content/14/3/543.full.html\#ref-list-1

License

Email Alerting Receive free email alerts when new articles cite this article - sign up in the box at the Service top right corner of the article or click here. 